\title{
Research on Public Satisfaction of Urban Management Based on AHP-fuzzy Comprehensive Evaluation
}

\author{
Yunxi Chen ${ }^{1, a, *}$ and Peng Wang ${ }^{1, b}$ \\ ${ }^{1} X i$ ' an University of Architecture and Technology, School of Management, Xi'an, Shanxi, P.R.China \\ a793016090@qq.com, b870383197@qq.com \\ *corresponding author
}

Keywords: Urban Management, Public Satisfaction, Analytic Hierarchy Process, Fuzzy Comprehensive Evaluation

\begin{abstract}
In urban management, public satisfaction represents a certain degree of management level and management effectiveness. In order to improve the public satisfaction of urban management, this paper studies the existing problems of public satisfaction in urban management in Xi'an and builds a comprehensive evaluation model to include various influencing factors. Divide into different levels, and use the consistent matrix method to compare the low-level factors to the high-level factors and get the relative importance. Then use the fuzzy comprehensive evaluation method to cycle from the lower level to the highest level. Finally, calculate the score and calculate the score according to the interval Give assessments and improvement measures and suggestions.
\end{abstract}

\section{基于 AHP-模糊综合评价的城市管理公众满意度研究}

\author{
陈芸茜 $1, \mathrm{a}, *$, 王鹏 $1, \mathrm{~b}$ \\ 1 西安建筑科技大学, 西安, 陕西, 中国 \\ a793016090@qq.com, b870383197@qq.com \\ *通讯作者
}

关键词：城市管理、公众满意度、层次分析法、模糊综合评价

摘要: 在城市管理中, 公众满意度在一定程度上代表了管理水平和管理效果, 针对如何提高城 市管理公众满意度，本文通过研究西安市城市管理公众满意现存问题，构建综合评价模型将各 种影响因素分为不同的层级, 并运用一致矩阵法将低层因素向高层因素两两相互对比得出相对 重要性, 再运用模糊综合评价法从低层向最高层循环运算, 最后计算出得分并根据分数所在区 间给出评价及改进措施和建议。

\section{1. 引言}

随着城市化的发展，城市管理复杂性急剧增加。城市管理的目的是城市健康发展，环境 绿色生态，人民安居乐业，但是，在现有的城市管理中，由于政府有时采取了 “单一导向” 的 管理模式，使政府和公众之间缺乏有效沟通，公众成为被动接受方，实际需求无法得到满足， 城市管理的效果得不到保障。这样的管理模式将导致城市管理成本虚高、效率低下，加深了公 众宜居环境需求日益增加和城市管理动能不足之间的矛盾, 最终引发公众对城市管理工作的不 满。在城市管理中，市民是城市发展最直接和最广泛的参与者，随着生活水平的提高，市民对 城市管理的要求和需求都提高了，良好的城市管理应以公众满意为导向。但是公众满意度受各 
种因素的影响，主观因素复杂，难以量化分析。本文构建 AHP-模糊综合评价模型将各种影响 因素分为不同的层级, 经过模糊综合满意度分析进行评价, 根据评价结果提出改进建议, 改善 现有公众满意度较低的现状，最后通过实例验证模型的实用性。

\section{2. 城市管理公众满意现状}

怎样更加有效的提高政府服务的公众满意度,是民主行政改革的重要目标,也是政府服务的 公众满意度研究的最终目标。但是随着城市化进程的加快, 城市管理的步伐显得缓慢, 由此导 致了公众对城市管理产生了诸多不满。主要体现在以下几个方面。

\section{1 公众对城市环境存在不满}

城市环境主要分为自然环境、生活环境、政策环境三方面。对于自然环境，近年来由于工 业的发展和私家车的普及, 西安市居民饱受雾皬的危害, 甚至有的小区饮用水浑浊等情况屡见 不鲜。同时, 城市绿化因此也受到影响, 长期雾䨪和灌溉用水的污染, 绿植无法正常进行生长。 关于生活环境，居民住房紧张，房价贵；医疗设施共享不均等，医疗资源分布不均等；受到古 城限制, 道路交通条件差, 经常发生拥堵情况。政策环境下, 居民参与城市管理得不到法律保 障，甚至无从参与，群众对城市管理制度也不够了解。

\section{2 公众对城市行政执法存在不满}

目前，城市管理执法体制不完善，城管执法制度缺失。城管执法局依据《行政处罚法》第 16 条设立以来，还未拥有属于界定城市管理权利义务的法律文件，城管进行执法活动只能依 据政府的授权，也就是说城管执法局实际处于无法可依的境地。执法程序不健全，最突出的问 题是执法人员谋私受贿问题。随着地方政府扩大城管的执法权限，城管执法部门的工作覆盖面 越来越大，同时监督和制约机制等配套措施跟不上，造成了执法人员贪污受贿等行为，工作不 被公众接受更谈不上满意。

其次是执法程序不规范。在实际执法活动中, 暴力执法是最为公众难以接受的, 还有在行 政执法不按照执法程序，有时甚至倒置执法程序以达到解决问题的办法，有时对相同事件做不 同处理，难以服众，很难做到令公众满意。

\section{3 公众对政府服务存在不满}

政府服务是公众直接接触政府的窗口，但往往其服务质量不能使公众满意。其主要体现在 工作效率低，办事群众等待时间过长；服务态度差，公务员缺乏服务意识，网络上曝光的很多 纠纷都是政府服务人员态度恶劣造成的; 政府办事程序不公开，导致群众 “花时间、白跑一趟” 的现象屡见不鲜。

解决以上导致公众对城市综合管理不满意的问题, 一方面政府应加强对城市综合管理的管 理力度，促进相关法律法规的制定落实，另一方面政府应将公众的满意度考虑进城市综合管理 工作中，进而提高公众对城市综合管理工作成果的认可。我们考虑可以使用 AHP 模糊综合评 价对目前公众满意度水平进行评价，根据最后的评价结果提出针对性建议。

\section{3. 城市管理满意度评价指标体系}

首先建立层次结构模型，将决策的目标、考虑的因素（决策准则）和决策对象按它们之间 的相互关系分为最高层、中间层和最低层，绘出层次结构图（见图 1)。 


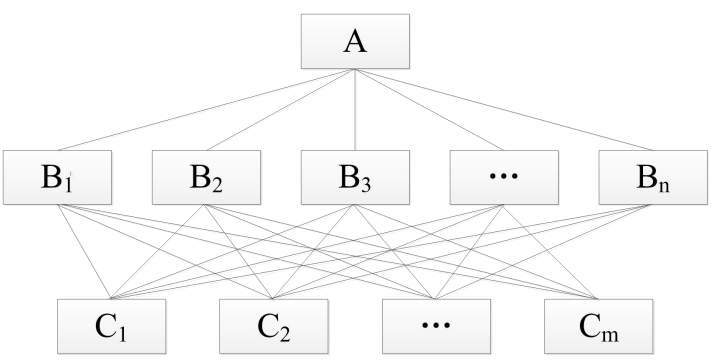

图 1 结构层次图

最高层: 即目标层。表示解决问题的目的, 即层次分析要达到的总目标。通常只有一个总 目标。

中间层：即准则层、指标层。表示采取某种措施、政策、方案等实现预定总目标所涉及的 中间环节。

最低层: 方案层。表示将选用的解决问题的各种措施、方案等。通常有几个方案可选。 根据国内城市管理公众满意度现状建立层次结构模型（见表 1） 
表 1 城市管理公众满意度影响因素层次模型

\begin{tabular}{|c|c|c|c|}
\hline 目标层 & 一级指标 & 二级指标 & 指标解释 \\
\hline \multirow{10}{*}{ 环境层面 } & \multirow{4}{*}{$\begin{array}{l}\text { 生活环境 } \\
\text { (B1) }\end{array}$} & 住房状况（ $C_{11} ）$ & 房地产平均价格水平 \\
\hline & & 医疗设施（ $C_{12} ）$ & 每千人拥有医院床位数 \\
\hline & & 交通道路条件（ $C_{13} ）$ & 每千人拥有医院床位数 \\
\hline & & 交通车辆状况（ $C_{14} ）$ & 每万人拥有公共汽车车辆 \\
\hline & \multirow{4}{*}{$\begin{array}{l}\text { 自然条件 } \\
\text { (B2) }\end{array}$} & 空气质量 $\left(C_{21}\right)$ & $\begin{array}{c}\text { 年内好于国家二级（良好）标准以 } \\
\text { 上的天数 }\end{array}$ \\
\hline & & 水源质量 $\left(C_{22}\right)$ & 工业废水排放达标率 \\
\hline & & 城市绿化（ $C_{23} ）$ & 建成区绿化覆盖率 \\
\hline & & 政府信息公开度 $\left(C_{31}\right)$ & 每月政府网站信息及时更新占比 \\
\hline & \multirow{2}{*}{$\begin{array}{l}\text { 政策环境 } \\
\text { (B3) }\end{array}$} & $\begin{array}{c}\text { 公众参与权利法律保障程度 } \\
\left(C_{32}\right)\end{array}$ & 相关法律法规数量 \\
\hline & & $\begin{array}{c}\text { 公众了解城市管理制度程度 } \\
\left(C_{33}\right)\end{array}$ & 每百人了解城市管理制度的人数 \\
\hline \multirow{7}{*}{ 社会层面 } & \multirow{2}{*}{$\begin{array}{l}\text { 信息传播 } \\
\text { (B4) }\end{array}$} & 新闻 $\left(C_{41}\right)$ & \multirow{7}{*}{$\begin{array}{c}\text { 城市管理相关新闻平均播出时长 } \\
\text { 城市管理相关词条搜索次数 } \\
\text { 在岗位职工参加各类保险的比例 } \\
\text { 近两年刑事案件恋数下降率 } \\
\text { 每百人关注人数 } \\
\text { 每百人关注人数 } \\
\text { 平均等待时间 }\end{array}$} \\
\hline & & 网络 $\left(C_{42}\right)$ & \\
\hline & 社会保障 & 社会保险覆盖率 $\left(C_{51}\right)$ & \\
\hline & (B5) & 社会安全程度（ $C_{52} ）$ & \\
\hline & \multirow{3}{*}{$\begin{array}{l}\text { 公民意识 } \\
\quad \text { (B6） }\end{array}$} & 公民的权利意识（ $C_{61} ）$ & \\
\hline & & 公民的责任意识（ $C_{62}$ ) & \\
\hline & & 办事效率（ $C_{71}$ ） & \\
\hline \multirow{9}{*}{ 公众层面 } & 服务质量 & 服务态度（ $C_{72} ）$ & 每月服务态度问题投诉次数 \\
\hline & 感知 (B7) & 办事程序公开度 $\left(C_{73}\right)$ & 每百人了解政府办事程序 \\
\hline & & 创新工作（ $C_{74} ）$ & 简便程序、创新工作设点数量 \\
\hline & \multirow{4}{*}{$\begin{array}{l}\text { 参与意愿 } \\
\text { (B8) }\end{array}$} & $\begin{array}{c}\text { 对城市更新项目的参与意愿 } \\
\left(C_{81}\right)\end{array}$ & $\begin{array}{c}\text { 每百人愿意参与到城市更新项目中 } \\
\text { 的人数 }\end{array}$ \\
\hline & & $\begin{array}{c}\text { 对城市行政执法的参与意愿 } \\
\left(C_{82}\right)\end{array}$ & $\begin{array}{c}\text { 每百人愿意参与到城市行政执法中 } \\
\text { 来的人数 }\end{array}$ \\
\hline & & $\begin{array}{c}\text { 对城市环境管理的参与意愿 } \\
\left(C_{83}\right)\end{array}$ & $\begin{array}{c}\text { 每百人愿意参与到环境管理中的人 } \\
\text { 数 }\end{array}$ \\
\hline & & 对城市环境的期望（ $\left.C_{91}\right)$ & $\begin{array}{c}\text { 每月对城市环境提出建议的热线电 } \\
\text { 话数量 }\end{array}$ \\
\hline & \multirow[t]{2}{*}{$\begin{array}{l}\text { 公众期望 } \\
\text { (B9） }\end{array}$} & 对更新项目的期望（ $C_{92}$ ) & $\begin{array}{c}\text { 每月对更新项目建议的热线电话数 } \\
\text { 量 }\end{array}$ \\
\hline & & 对城市执法的期望（ $\left.C_{93}\right)$ & $\begin{array}{c}\text { 每月对城市环境提出建议的热线电 } \\
\text { 话数量 }\end{array}$ \\
\hline
\end{tabular}

\section{4. 评价模型及实证研究}

\section{1 评价模型}

在层次分析法的基础上构建模糊综合评价，两者的结合运用提高了评价结果的有效性与可靠 性, 步骤如下。

(1) 建立层次结构模型 通过 Saaty 的一致矩阵法构造判断矩阵, 形成一个定性结果。 
设要比较各准则 $B_{1}, B_{2}, \cdots, B_{\mathrm{n}}$ 对目标 $A$ 的重要性, $B_{i}: B_{j}=a_{i j} ; A=\left(a_{i j}\right)_{n \times n}$, $a_{i j>0,} a_{i j}=1 / a_{i j}$, 给出判断矩阵: $A_{\mathrm{n} \times n}$

(2) 计算单排序权向量并做一致性检验

对应于判断矩阵最大特征根 $\lambda$ max 的特征向量, 经归一化后记为权向量 $W$ 。

定义一致性指标： $C I=0$ ，有完全的一致性 $C I$ 接近于 0 , 有满意的一致性; $C I$ 越大，不 一致越严重。

引入判断矩阵的平均随机一致性指标 $R I$ 值, 当阶数 $n>2$ 时, 判断矩阵一致性指标 $C I$ 与同 阶平均随机性指标 $R I$ 的比值为判断矩阵的随机一致性比率记为 $C R$, 即 $C R=C I / R I$ 。

定义一致性率比: $C R=\frac{C I}{R I}$, 当 $\frac{C I}{R I} \leq 0.1$ 时, 认为 $A$ 的不一致程度在容许范围之内, 有满 意的一致性, 通过一致性检验。可用其归一化特征向量作为权向量, 记为 $W$ 。否则要重新构 造成对比较矩阵 $A$, 对 $a_{i j}$ 加以调整。

(3) 层次总排序及其一致性检验。

$B$ 层 $B_{1}, B_{2}, \cdots, B_{n}$ 对总目标 $A$ 的排序为 $b_{1}, b_{2}, \cdots, b_{n}, C$ 层 $C_{1}, C_{2}, \cdots, C_{m}$ 对上层 $B$ 中因素 $B_{j}(j=1,2, \ldots, n)$ 的单次排序为 $C_{1 j}, C_{2 j}, \cdots, C_{m j} \quad(j=1,2, \ldots, n)$ 。

一致性比率为: $C R=\frac{b_{1} C I_{1}+b_{2} C I_{2}+\ldots b_{n} C \mathrm{Im}_{\mathrm{m}}}{b_{1} R I_{1}+b_{2} R I_{2}+\ldots+b_{n} R \mathrm{I}_{\mathrm{m}}}$

当 $C R<0.1$ 时, 认为层次总排序通过一致性检验。

(4) 确定评价因素集合 $U=\left\{u_{1}, u_{2}, \ldots, u_{n}\right\}$ 。

其中 $U_{i}\left\{u_{1}, u_{2}, \ldots, u_{n}\right\}$ 为评价因素， $n$ 是同一层次上单个因素的个数，这一集合构成了评价 的框架。

(5) 确定公众满意度等级标准集合 $V=\left\{v_{1}, v_{2}, \ldots, v_{n}\right\}$

其中 $V_{i}\left\{v_{1}, v_{2}, \ldots, v_{n}\right\}$ 是评价等级标准, $n$ 是元素个数, 即等级数或评语档次数。

本文将城市管理公众满意度分为满意度高、满意度较高、满意度中等、满意度较低、满意 度低五个等级, 公众满意度评判集: $V=\left\{v_{1}, v_{2}, \ldots, v_{n}\right\}=\{$ 高, 较高, 中等, 较低, 低 $\}$, 其等 级分值对应赋为 $E=\{90,80,70,60,50\}$ 。

(6) 确定隶属度矩阵

$$
R=\left[\begin{array}{c}
R_{1} \\
R_{2} \\
\ldots \\
R_{n}
\end{array}\right]=\left[\begin{array}{cccc}
r_{11} & r_{12} & \ldots & r_{15} \\
r_{21} & r_{22} & \ldots & r_{25} \\
\ldots & \ldots & \ldots & \ldots \\
r_{n 1} & r_{n 2} & \ldots & r_{n 5}
\end{array}\right]
$$

$R_{i}=\left(r_{i}, r_{i 2}, \ldots, r_{i n}\right)$ 是对第 $i$ 个评价因素 $u_{i}$, 进行单因素评价得到一个相对于 $V_{j}$ 的模糊向量。

$r_{i j}$ 表示第 $i$ 个因素 $u_{i}$ 对公众满意度等级 $V_{j}$ 的隶属度, $0<r_{i j}<1$ 。

(7) 模糊综合满意度评价

$$
B i=\left(W_{C 1}, W_{C 2}, \ldots, W_{C m}\right) \bullet\left[\begin{array}{cccc}
r_{11} & r_{12} & \ldots & r_{1 m} \\
r_{21} & r_{22} & \ldots & r_{2 m} \\
\ldots & \ldots & \ldots & \ldots \\
r_{n 1} & r_{n 2} & \ldots & r_{m 5}
\end{array}\right]=R_{i} ;
$$

$W_{C i}=\left\{W_{C 1}, W_{C 2}, \ldots, W_{C m}\right\}$ 为 $C$ 层指标权重, 其中 $0 \leq W_{C i} \leq 1, \sum_{i=1}^{m} W_{C i}=1$ 。

$A$ 层综合评价值集 $A=\left(W_{B 1}, W_{B 2}, \ldots, W_{B n}\right) \bullet\left(R_{1}, R_{2}, \ldots, R_{n}\right)^{T}$; 
将再将满意度评判集 $E$ 代入, 综合计算出该地区的整体公众满意度得分 $F=A \bullet E$ 。

\section{2 基于西安市城市管理公众满意度的实证分析}

根据层次分析法计算得出权重

$$
\begin{aligned}
& W_{C 1}=(0.2496,0.2274,0.3224,0.2006) ; \quad W_{C 2}=(0.3333,0.5450,0.1217) ; \\
& W_{C 3}=(0.3333,0.3066,0.3601) ; \quad W_{C 4}=(0.3672,0.6328) ; \\
& W_{C 5}=(0.6992,0.3008) ; \quad W_{C 6}=(0.3501,0.6499) ; \\
& W_{C 7}=(0.2879,0.3387,0.2044,0.1690) ; \quad W_{C 8}=(0.3333,0.2477,0.4190) ; \\
& W_{C 9}=(0.3334,0.2326,0.4340)
\end{aligned}
$$

邀请城市管理领域的 10 位专家对各因素进行评价，得出准则层的模糊评价矩阵如下：

$$
\begin{aligned}
& R_{1}=\left[\begin{array}{ccccc}
0.1 & 0.2 & 0.4 & 0.2 & 0.1 \\
0 & 0.1 & 0.2 & 0.4 & 0.3 \\
0.1 & 0.3 & 0.3 & 0.2 & 0.1 \\
0 & 0.1 & 0.4 & 0.3 & 0.2
\end{array}\right] \quad R_{2}=\left[\begin{array}{ccccc}
0.1 & 0.4 & 0.3 & 0.1 & 0.1 \\
0.1 & 0.5 & 0.2 & 0.1 & 0.1 \\
0.5 & 0.3 & 0.1 & 0.1 & 0
\end{array}\right] \\
& R_{3}=\left[\begin{array}{ccccc}
0.1 & 0.1 & 0.5 & 0.2 & 0.1 \\
0 & 0.1 & 0.1 & 0.3 & 0.5 \\
0 & 0.1 & 0.2 & 0.2 & 0.5
\end{array}\right] \quad R_{4}=\left[\begin{array}{ccccc}
0 & 0.1 & 0.2 & 0.3 & 0.4 \\
0.4 & 0.2 & 0.2 & 0.1 & 0.1
\end{array}\right] \\
& R_{5}=\left[\begin{array}{lllll}
0.3 & 0.2 & 0.2 & 0.2 & 0.1 \\
0.4 & 0.3 & 0.1 & 0.1 & 0.1
\end{array}\right] \quad R_{6}=\left[\begin{array}{lllll}
0.5 & 0.2 & 0.1 & 0.1 & 0.1 \\
0.1 & 0.1 & 0.2 & 0.3 & 0.3
\end{array}\right] \\
& R_{7}=\left[\begin{array}{lllll}
0.1 & 0.3 & 0.2 & 0.2 & 0.2 \\
0.3 & 0.2 & 0.2 & 0.2 & 0.1 \\
0.4 & 0.3 & 0.1 & 0.1 & 0.1 \\
0.2 & 0.2 & 0.2 & 0.2 & 0.2
\end{array}\right] \quad R_{8}=\left[\begin{array}{lllll}
0.1 & 0.2 & 0.3 & 0.2 & 0.2 \\
0.3 & 0.3 & 0.2 & 0.1 & 0.1 \\
0.3 & 0.2 & 0.2 & 0.2 & 0.1
\end{array}\right] \\
& R_{9}=\left[\begin{array}{lllll}
0.4 & 0.3 & 0.1 & 0.1 & 0.1 \\
0.2 & 0.2 & 0.2 & 0.2 & 0.2 \\
0.5 & 0.2 & 0.1 & 0.1 & 0.1
\end{array}\right]
\end{aligned}
$$

利用 Matlab7.0 编程计算求得：目标层的模糊评价矩阵为

$$
R=\left[\begin{array}{l}
R_{1} \\
R_{2} \\
R_{3} \\
R_{4} \\
R_{5} \\
R_{6} \\
R_{7} \\
R_{8} \\
R_{9}
\end{array}\right]=\left[\begin{array}{l}
B_{1} \\
B_{2} \\
B_{3} \\
B_{4} \\
B_{5} \\
B_{6} \\
B_{7} \\
B_{8} \\
B_{9}
\end{array}\right]=\left[\begin{array}{lllll}
0.0572 & 0.1894 & 0.3223 & 0.2655 & 0.1655 \\
0.1487 & 0.4423 & 0.2212 & 0.1000 & 0.0878 \\
0.0333 & 0.1000 & 0.2693 & 0.2307 & 0.3667 \\
0.2531 & 0.1633 & 0.2000 & 0.1734 & 0.2102 \\
0.3301 & 0.2301 & 0.1699 & 0.1699 & 0.1000 \\
0.2400 & 0.1350 & 0.1650 & 0.2300 & 0.2300 \\
0.2460 & 0.2492 & 0.1796 & 0.1796 & 0.1457 \\
0.2333 & 0.2248 & 0.2333 & 0.1752 & 0.1333 \\
0.3969 & 0.2333 & 0.1233 & 0.1233 & 0.1233
\end{array}\right]
$$

$A=\left(W_{B 1}, W_{B 2}, \ldots, W_{B 9}\right) \bullet\left(R_{1}, R_{2}, \ldots, R_{9}\right)=[0.2001,0.2193,0.2197,0.1864,0.1745]$; 则

$F=A \bullet E=70.8410$ 


\section{5. 结论及建议}

通过 AHP-模糊综合评价法对西安市城市管理公众满意情况计算出的模糊综合评分为

70.8410, 说明西安市公众满意度水平贴近中等水平。

根据西安市的实例数据, 可以看出影响公众对城市管理的满意度最明显的是公众层面的公 众感知质量, 其权重为 $14.97 \%$, 是 9 个一级指标中最重要的。在其下一级指标中, 所占权重 最大的对城市行政执法的感受，权重占到了其上级指标的 $33.87 \%$ 。在提高城市管理公众满意 度时, 应着重从占比较高的几个因素入手。通过专家调查的数据整理, 发现导致西安市城市管 理公众满意度不佳的原因主要集中在政府形象欠佳、管理体制不顺畅、服务质量不高、信息不 对称。对此, 笔者提出四点建议:

一是加强城市管理综合执法部门的形象管理。强化执法者的职业道德建设, 做到公正执法、 文明执法。强化城市管理部门的队伍建设, 促进办事效率, 文明办事方式。在面貌上, 配备统 一的装备, 并且对城市管理综合执法部门进行形象设计与宣传, 合理利用信息化的平台, 微信、 微博等做正面宣传。培养城市管理部门舆论危机应对能力，有效应对负面报道。

二是，在城管行政执法部门继续加强和完善问责制度。只有将责任具体到执行的各个环节， 各个环节的负责人及主要责任人，才能真起到问责的作用。我国城管行政执法一些领域中，还 存在着多头执法的现象, 其实, 随着我国社会的发展, 各种现象的存在与发生趋复杂, 牵连的 领域很多，执法领域难免存在交叉的问题。但是，当责任发生时却出现了对自己有利的，各部 门争相管理, 不利的相互推诿的现象。因此, 需要对责任进行进一步的完善和量化。在存在城 管行政执法权力交叉的领域，可以实行连带责任制度。这种规定，可以使城管各部门在执法交 叉的领域更好的互动与协调，妥善的解决好矛盾。

三是, 引入市民满意度测评衡量部门绩效, 以此督促部门提高服务质量。城市管理部门考 核办法一个重要的特点是业务工作与各种特殊人群密切相关,如城管处、街道办,这样的业务处 室可以引入市民满意度测评来衡量部门绩效, 通过电话调查或填写满意度测评表的形式调查被 服务人群接受公共服务的经历和他们的满意度(预期、实际表现、满意程度、改进)。虽然公众 的评判有种种缺陷:也许缺乏评估的专门技术、知识,也许不了解政府的实际运作,也许缺乏必要 的准确的信息,但城市管理部门考核办法本身就是为民众而存在,政府绩效就应以公益为参照坐 标,树立公民取向的绩效观,接受公众监督,对那些内设处室如办公室、人事处、党团委、审计处 等服务性处室为顾客进行满意度测评。部门绩效测评结果将作为改进服务质量的依据。

四是建立与公众沟通的多方途径, 突破城市管理信息不对称的瓶颈。公众不仅是城市管理 成果的享用者, 更是诚市管理的参与者。城市管理不仅是城市管理信息的发布者, 更是公众心 声的倾听者。传统城市管理往往过于注重作为管理者信息的发布，而对市民公众信息的反馈收 集不够，在 “官”与 “民”之间形成了信息不对称。在城市管理当局和市民之间应该建立起有 效的信息反馈渠道，形成双向的信息交流，良性互动。这不但是管理当局获取有用信息的有效 渠道, 更是提高城市管理公众理解度的有效途径。对此, 我们可以借鉴新加坡的做法来改善我 市城市管理者与公众的沟通交流状况。

本文满意度因素指标体系是通过参考大量的文献，总结现有研究成果建立的，带有一定主 观性, 未来的研究可通过专家打分法来进行指标体系的笁选, 使指标体系更客观。城市管理公 众满意度评价中评价等级的确定带有一定的主观性, 未来的研究中最好能够找到能替代的定量 指标并加以量化。

\section{参考文献}

[1] 李洪涛. 城市居民的社区满意度及其对社区归属感的影响[D].华中科技大学,2005.

[2] 杨蓓蕾. 面向发展质量的城市社区治理研究[D].同济大学,2007.

[3] 耿金花,高齐圣.基于联立方程的社区满意度模型[J].系统工程,2007(03):111-114. 
[4] 应瑛,寿涌毅, 吴晓波.城市管理公众满意度指数模型实证分析 [J]. 城市发展研 究,2009,16(01):102-108+119.

[5] 肖谢.城市管理路径探究——基于公众参与视角下的协管员制度 [J]. 安徽行政学院学 报,2015,6(03):99-104.

[6] 范柏乃,金洁.公共服务供给对公共服务感知绩效的影响机理一一政府形象的中介作用与公 众参与的调节效应[J].管理世界,2016(10):50-61+187-188.

[7] 周鹤群.公众参与:城市管理机制创新的重要方向[J].上海城市管理,2015,24(01):74-77.

[8] 唐礼勇,丁盛熔. 公众参与:城市管理综合行政执法困境破解的新探索 [J]. 领导科 学,2015(29):57-59. 